\title{
Clinical evaluation of sivelestat for acute lung injury/acute respiratory distress syndrome following surgery for abdominal sepsis
}

This article was published in the following Dove Press journal:

Drug Design, Development and Therapy

9 October 2012

Number of times this article has been viewed

\author{
Yoshiaki Tsuboko' \\ Shinhiro Takeda ${ }^{1,2}$ \\ Seiji Mii' \\ Keiko Nakazato' \\ Keiji Tanaka ${ }^{2}$ \\ Eiji Uchida ${ }^{3}$
}

Atsuhiro Sakamoto'

'Department of Anesthesiology, Nippon Medical School, ${ }^{2}$ Intensive Care Unit and Cardiac Care Unit, Nippon Medical School Hospital, ${ }^{3}$ Department of Surgery, Nippon Medical School, Tokyo, Japan
Correspondence: Yoshiaki Tsuboko I-I-5 Sendagi, Bunkyo-ku, Tokyo II3-8603, Japan

Tel +81358146243

$\mathrm{Fax}+81356853077$

Email tsubo-yossy@nms.ac.jp
Background: The efficacy of sivelestat in the treatment of acute lung injury/acute respiratory distress syndrome (ALI/ARDS) has not been established. In part, this is due to the wide variety of factors involved in the etiology of ALI/ARDS. In this study, we examined the efficacy of sivelestat in patients with ALI/ARDS associated with abdominal sepsis.

Methods: The subjects were 49 patients with ALI/ARDS after surgery for abdominal sepsis. The efficacy of sivelestat was retrospectively assessed in two treatment groups, ie, a sivelestat group $(\mathrm{n}=34)$ and a non-sivelestat group $(\mathrm{n}=15)$.

Results: The sivelestat group showed significant improvements in oxygenation, thrombocytopenia, and multiple organ dysfunction score. The number of ventilator days (6.6 \pm 6.1 versus $11.1 \pm 8.4$ days; $P=0.034)$ and length of stay in the intensive care unit $(8.5 \pm 6.2$ versus $13.3 \pm 9.5$ days; $P=0.036)$ were significantly lower in the sivelestat group. The hospital mortality rate decreased by half in the sivelestat group, but was not significantly different between the two groups.

Conclusion: Administration of sivelestat to patients with ALI/ARDS following surgery for abdominal sepsis resulted in early improvements of oxygenation and multiple organ dysfunction score, early ventilator weaning, and early discharge from the intensive care unit.

Keywords: sivelestat, acute lung injury, acute respiratory distress syndrome, abdominal sepsis

\section{Introduction}

Acute lung injury/acute respiratory distress syndrome (ALI/ARDS) is still associated with high mortality, despite advances in understanding of the etiology and treatment of the disorder, and is one of the main severe clinical conditions managed in the intensive care unit (ICU). ${ }^{1}$ Many randomized clinical trials of new drugs for treatment of ALI/ ARDS have been conducted, ${ }^{2-4}$ but most have had negative findings and there are no new effective therapeutic approaches. ${ }^{5}$

Sivelestat, a selective neutrophil elastase inhibitor, was found to be effective for reducing the length of ICU stay and the ventilator-assisted period for patients with ALI/ARDS in a multicenter clinical study conducted in Japan, ${ }^{6}$ but similar efficacy has not been found in other countries. ${ }^{7}$ This may in part be because ALI/ARDS is associated with a wide variety of underlying diseases, and the patient characteristics may be too heterogeneous for determination of therapeutic efficacy. Therefore, the therapeutic effect of sivelestat on ALI/ARDS requires evaluation in a population with more homogeneous etiology of the underlying disease. ${ }^{5}$

Sepsis is a major cause of extrapulmonary ALI/ARDS and is associated with high mortality. ${ }^{8}$ In patients with sepsis, marked accumulation of neutrophils in the lungs is 
found in the early stages. ${ }^{9,10}$ ALI/ARDS arising from abdominal sepsis is associated with lung tissue damage caused by neutrophil elastase released from activated neutrophils, active oxygen, or other factors. Because sivelestat selectively inhibits neutrophil elastase without having effects on other proteases, this drug may be effective in ALI/ARDS associated with abdominal sepsis. Therefore, we investigated the effects of sivelestat in the treatment of this condition.

\section{Materials and methods}

Data for all patients admitted to the ICU of Nippon Medical School Hospital with a diagnosis of ALI/ARDS following surgery for abdominal sepsis were analyzed retrospectively. The study period extended from January 1, 2002 to December 31, 2008. The ethical committee at our hospital has established that retrospective studies involving analysis of medical records can be conducted without authorization from the committee. An ICU physician made a diagnosis of ARDS on admission to the ICU and also made the decision whether to use sivelestat. Patients who did and did not receive sivelestat were defined as the sivelestat group and non-sivelestat group, respectively. Sivelestat infusion was started at $0.2 \mathrm{mg} / \mathrm{kg} /$ hour immediately after ICU admission and was continued until withdrawal from the ventilator or after 14 days, whichever was the shorter period.

In all cases, the origin of sepsis was abdominal and required laparotomy or drainage. All patients underwent abdominal surgery for abdominal sepsis before admission to ICU, and were directed to the ICU immediately. ALI/ARDS was defined according to the American-European Consensus Conference definition. ${ }^{11}$ Sepsis and septic shock were defined according to the criteria of the American College of Chest Physicians/Society of Critical Care Medicine Consensus Conference definition. ${ }^{12}$ The daily multiple organ dysfunction (MOD) score was also used in the evaluation. ${ }^{13}$ Patients with associated diagnoses, such as interstitial pneumonia, malignancy with a poor short-term prognosis, those with a do-not-resuscitate order on admission, and those with coma on admission were excluded. Coma was defined as a Glasgow Coma Scale score $<10$.

A clinical assessment (heart rate, blood pressure, level of consciousness, $\mathrm{SpO}_{2}$, respiratory rate) was performed regularly, as well as assessment of arterial blood gases, common laboratory data, and other variables $\left(\mathrm{PaO}_{2} / \mathrm{FiO}_{2}, \mathrm{PCO}_{2}\right.$, $\mathrm{pH}, \mathrm{C}$-reactive protein, platelet count). These data and the clinical history of the patients were obtained retrospectively from medical records. Information was collected for age, gender, primary diagnosis, surgical procedures, presence of diabetes mellitus, Glasgow Coma Scale score, Acute Physiology and Chronic Health Evaluation (APACHE) II score, and Simplified Acute Physiology Score (SAPS) II on ICU admission, and for outcomes including ICU mortality and inhospital mortality. Arterial blood gases, heart rate, and arterial blood pressure were obtained on the day of ICU admission and on postoperative days 1 and 2. Ventilator settings were also recorded.

A Student's $t$-test was performed to evaluate between-group differences in patient characteristics on admission to ICU. A two-way repeated-measures analysis of variance and the Bonferroni multiple comparisons procedure were performed to evaluate within-group and between-group time courses. A Mann-Whitney test was performed to evaluate ventilatorassisted days and length of ICU stay between groups. A Fisher's exact test or $\chi^{2}$ test was used to compare the ICU mortality rate and final inhospital mortality rate. All statistical analyses were performed using the SPSS II software program (Abacus Concepts, Berkeley, CA). All values are reported as the mean \pm standard deviation, and all $P$ values $<0.05$ were considered to be statistically significant.

\section{Results}

ARDS following surgery for abdominal sepsis occurred in 53 cases in the study period, including 49 cases judged to be eligible for inclusion in the study. The four excluded cases included one with interstitial pneumonia, one with cancerous peritonitis and a poor short-term prognosis, one with a donot-resuscitate order on admission, and a bedridden patient who was comatose on admission with a Glasgow Coma Scale score of 9 . Of the 49 eligible subjects, 34 received sivelestat and 15 did not receive sivelestat.

The patient characteristics listed in Table 1 show that $39 \%$ of cases were in shock during surgery or on admission to the ICU. Shock was present in $47 \%$ of patients in the sivelestat group and in $20 \%$ in the non-sivelestat group. No other variables differed significantly between the groups at the time of ICU admission (Table 1). Inotropic agents were used in all cases, with norepinephrine being the most frequently used agent $(89.8 \%)$. Vasopressin was used for three patients in the sivelestat group. Fifteen patients $(44 \%)$ in the sivelestat group and six $(40 \%)$ in the non-sivelestat group required renal replacement therapy, and seven $(21 \%)$ in the sivelestat group and three $(20 \%)$ in the non-sivelestat group received polymyxin B-immobilized fiber column hemoperfusion.

The patients had various preoperative medical histories (Table 2). The most common underlying disease was perforation of the colon, affecting 19 (55.8\%) patients in the 
Table I Patient characteristics on admission to the ICU

\begin{tabular}{llll}
\hline & Sivelestat & Non-sivelestat & P value \\
\hline Number of patients & 34 & 15 & \\
Age (years) & $73 \pm 9$ & $69 \pm 17$ & 0.84 \\
Sex (male/female) & $27 / 7$ & $10 / 5$ & 0.34 \\
APACHE II score & $22 \pm 7$ & $21 \pm 4$ & $0.5 \mathrm{I}$ \\
SAPS II & $49 \pm 12$ & $49 \pm 13$ & 0.95 \\
MOD score & $7.9 \pm 3.3$ & $7.3 \pm 3.0$ & 0.66 \\
Creatinine (mg/dL) & $1.7 \pm 1.6$ & $2.2 \pm 1.8$ & 0.87 \\
Diabetes mellitus, $\mathrm{n}(\%)$ & $4(12 \%)$ & $3(20 \%)$ & 0.45 \\
Renal replacement & $6(40 \%)$ & $15(44 \%)$ & 0.79 \\
therapy n, (\%) & & & \\
WBC $<4000, \mathrm{n}(\%)$ & $13(38 \%)$ & $5(33 \%)$ & 0.74 \\
CRP (mg/dL) & $2.36 \pm 0.93$ & $2.84 \pm 0.82$ & 0.89 \\
Shock, $\mathrm{n}(\%)$ & $16(47 \%)$ & $3(20 \%)$ & 0.07 \\
(SBP $<80$ mmHg) & & & \\
Inotropic agents & $34(100 \%)$ & $15(100 \%)$ & 1.00 \\
Initial ventilation mode & & & 0.55 \\
in ICU & & & \\
$\quad$ Pressure control, $\mathrm{n}(\%)$ & $27(79 \%)$ & $13(87 \%)$ & \\
$\quad$ Pressure support, $\mathrm{n}(\%)$ & $7(21 \%)$ & $2(13 \%)$ & 0.33 \\
Initial ventilatory pressure & & & \\
$\quad$ PEEP level (cm $\left.\mathrm{H}_{2} \mathrm{O}\right)$ & $\mathrm{II} .4 \pm 2.5$ & $10.7 \pm 2.2$ \\
$\quad$ PIP level (cm $\left.\mathrm{H}_{2} \mathrm{O}\right)$ & $22.7 \pm 4.0$ & $21.9 \pm 4.1$ & \\
\hline N & &
\end{tabular}

Note: Data are shown as the mean $\pm S D$, or number $(n)$ of patients.

Abbreviations: ICU, intensive care unit; APACHE, acute physiology and chronic health evaluation; SAPS, simplified acute physiology score; MOD score, multiple organ dysfunction score; WBC, white blood cell; CRP, C-reactive protein; SBP, systolic blood pressure; PEEP, positive end expiratory pressure; PIP, peak inspiratory pressure.

sivelestat group and $10(66.7 \%)$ in the non-sivelestat group; however, the difference was not statistically significant. Preoperative complications were present in 15 patients (44\%) in the sivelestat group and six $(40 \%)$ in the non-sivelestat group; however, the difference was not significant (Table 2).

$\mathrm{PaO}_{2} / \mathrm{FiO}_{2}$ improved significantly during treatment in both groups, but with a more significant improvement noted in the sivelestat group (Table 3). Patients in the sivelestat group had lower mean arterial pressure at baseline and showed significant improvement over time. Heart rate was similar in the two groups at baseline and showed a greater decrease over time in the sivelestat group. The MOD score was also similar at baseline and decreased significantly in the sivelestat group by postoperative day 2 , while remaining unchanged in the non-sivelestat group. The platelet count was stable in the sivelestat group, but was significantly decreased in the non-sivelestat group.

Ventilator management was undertaken based on the lung protective strategy designed by the ARDS network. ${ }^{14}$ The ventilation-assisted period and length of ICU stay were significantly shorter in the sivelestat group (Table 4), with both being about 5 days shorter than in the non-sivelestat group. The ICU and inhospital mortalities were about two-fold higher in the
Table 2 Diagnosis, surgical procedure, and preoperative complications

\begin{tabular}{|c|c|c|c|}
\hline & Sivelestat & Non-sivelestat & \\
\hline \multicolumn{4}{|l|}{ Diagnosis } \\
\hline Colon perforation & $19(55.9 \%)$ & $10(66.7 \%)$ & $P=0.69$ \\
\hline $\begin{array}{l}\text { Ascending, trans, or } \\
\text { descending colon }\end{array}$ & $9(26.5 \%)$ & $2(13.3 \%)$ & \\
\hline Sigmoid colon & $8(23.5 \%)$ & $5(33.3 \%)$ & \\
\hline Rectum & $2(5.9 \%)$ & $3(20.0 \%)$ & \\
\hline Intestinal perforation & I (2.9\%) & I (6.7\%) & \\
\hline Intestinal perforation + ileus & I (2.9\%) & I (6.7\%) & \\
\hline $\begin{array}{l}\text { Intestinal perforation }+ \\
\text { pancreatic leak }\end{array}$ & $0(0 \%)$ & I (6.7\%) & \\
\hline Intestinal necrosis + ileus & I (2.9\%) & $0(0 \%)$ & \\
\hline lleus & 7 (20.6\%) & $0(0 \%)$ & \\
\hline Abscess & $0(0 \%)$ & I (6.7\%) & \\
\hline Esophageal perforation & I (2.9\%) & $0(0 \%)$ & \\
\hline Necrotizing cholecystitis & I (2.9\%) & $0(0 \%)$ & \\
\hline Perforative biliary peritonitis & I (2.9\%) & I (6.7\%) & \\
\hline Ischemic enterocolitis & I (2.9\%) & $0(0 \%)$ & \\
\hline Ischemic colitis & I (2.9\%) & $0(0 \%)$ & \\
\hline \multicolumn{4}{|l|}{ Surgical procedure } \\
\hline Colectomy and/or colostomy & 27 (79.4\%) & II (73.3\%) & \\
\hline Colectomy and colostomy & $15(44.1 \%)$ & 7 (46.7\%) & \\
\hline Colectomy & $4(\mathrm{II} .8 \%)$ & $2(13.3 \%)$ & \\
\hline Hartmann's operation & $8(23.5 \%)$ & $2(13.3 \%)$ & \\
\hline Small intestinal resection & $2(5.9 \%)$ & $2(13.3 \%)$ & \\
\hline Ileocecal resection & $2(5.9 \%)$ & $0(0 \%)$ & \\
\hline Drainage & I (2.9\%) & I (6.7\%) & \\
\hline Pancreas total resection & $0(0 \%)$ & I (6.7\%) & \\
\hline $\begin{array}{l}\text { Cholecystectomy, liver } \\
\text { resection }\end{array}$ & I (2.9\%) & $0(0 \%)$ & \\
\hline Cholecystectomy & I (2.9\%) & $0(0 \%)$ & \\
\hline Preoperative & & & $P=0.96$ \\
\hline \multicolumn{4}{|l|}{ complications: past history } \\
\hline Abdominal aortic aneurysm & I (2.9\%) & I (6.7\%) & \\
\hline Antiphospholipid syndrome & I (2.9\%) & $0(0 \%)$ & \\
\hline Atrial fibrillation & I (2.9\%) & $0(0 \%)$ & \\
\hline Cerebrovascular accident & $3(8.8 \%)$ & $0(0 \%)$ & \\
\hline Chronic renal failure & I (2.9\%) & I (6.7\%) & \\
\hline Esophagectomy & I (2.9\%) & $0(0 \%)$ & \\
\hline Gastrectomy & I (2.9\%) & $0(0 \%)$ & \\
\hline Ischemic heart disease & $2(5.9 \%)$ & $2(13.3 \%)$ & \\
\hline Mitral valve replacement & I (2.9\%) & $0(0 \%)$ & \\
\hline Multifocal liver-kidney cysts & I (2.9\%) & $0(0 \%)$ & \\
\hline Pheochromocytoma & $0(0 \%)$ & I (6.7\%) & \\
\hline Pulmonary thromboembolism & I (2.9\%) & $0(0 \%)$ & \\
\hline Rheumatic arthritis & $0(0 \%)$ & I (6.7\%) & \\
\hline Ulcerative colitis & I (2.9\%) & $0(0 \%)$ & \\
\hline
\end{tabular}

Notes: Number (n) of patients (\%); 34 patients in the sivelestat group, 15 patients in the non-sivelestat group.

non-sivelestat group, but the difference for the sivelestat group was not significant. In the non-sivelestat group, one patient died of hemoperitoneum and multiorgan failure, one died of respiratory failure, and one died of leakage and multiorgan failure. In the sivelestat group, one patient died of leakage and multiorgan failure and three died of multiorgan failure. 
Table 3 Changes in blood gas values, hemodynamics, platelets, and MOD score

\begin{tabular}{|c|c|c|c|c|}
\hline & Group & ICU admission & POD I & POD 2 \\
\hline \multirow[t]{2}{*}{$\mathrm{pH}$} & Sivelestat & $7.35 \pm 0.10$ & $7.42 \pm 0.06 * *$ & $7.44 \pm 0.06 * *$ \\
\hline & Non-sivelestat & $7.36 \pm 0.07$ & $7.4 I \pm 0.07$ & $7.41 \pm 0.05^{\dagger}$ \\
\hline $\mathrm{PaO}_{2} / \mathrm{FiO}_{2}$ & Sivelestat & $|7| \pm 62$ & $288 \pm 89 * *$ & $294 \pm 79 * *$ \\
\hline$(\mathrm{mmHg})$ & Non-sivelestat & $182 \pm 65$ & $228 \pm 70^{*, \dagger}$ & $246 \pm 61 * *, \dagger$ \\
\hline $\mathrm{PaCO}_{2}$ & Sivelestat & $42 \pm 11$ & $37 \pm 6 * *$ & $37 \pm 6^{* *}$ \\
\hline$(\mathrm{mmHg})$ & Non-sivelestat & $37 \pm 7$ & $37 \pm 9$ & $38 \pm 5$ \\
\hline Heart rate & Sivelestat & $119 \pm 18$ & $95 \pm 18 * *$ & $85 \pm 16^{* *}$ \\
\hline (bpm) & Non-sivelestat & $119 \pm 12$ & $\mathrm{III} \pm 9 *,+\dagger$ & $101 \pm 1 \mid * *,+t$ \\
\hline Mean blood pressure & Sivelestat & $68 \pm 23$ & $86 \pm 12 * *$ & $88 \pm 13 * *$ \\
\hline$(\mathrm{mmHg})$ & Non-sivelestat & $81 \pm 16^{\dagger}$ & $84 \pm 15$ & $85 \pm 13$ \\
\hline CVP & Sivelestat & $11.8 \pm 3.3$ & $11.3 \pm 3.1$ & $9.0 \pm 3.4$ \\
\hline$(\mathrm{mmHg})$ & Non-sivelestat & $12.1 \pm 5.4$ & $11.6 \pm 3.4$ & $10.1 \pm 3.5$ \\
\hline Platelet & Sivelestat & $13.4 \pm 7.7$ & $12.4 \pm 6.8$ & $13.4 \pm 7.4$ \\
\hline$\left(10^{4} / \mu \mathrm{L}\right)$ & Non-sivelestat & $14.7 \pm 9.8$ & $1 \mathrm{I} .5 \pm 8.4$ & $9.1 \pm 8.3^{t \dagger}$ \\
\hline \multirow[t]{2}{*}{ MOD score } & Sivelestat & $7.9 \pm 3.3$ & $4.8 \pm 3.0^{* *}$ & $4.5 \pm 3.3^{* *}$ \\
\hline & Non-sivelestat & $7.3 \pm 3.0$ & $7.1 \pm 2.9^{\dagger}$ & $6.5 \pm 3.4^{\dagger}$ \\
\hline
\end{tabular}

Notes: Data are shown as the mean \pm standard deviation. 34 patients in the sivelestat group, I5 patients in the non-sivelestat group. $* P<0.05 ; * * P<0.0$ I versus ICU admission. ${ }^{\dagger} P<0.05$; ${ }^{\dagger} P<0.01$ for sivelestat group versus non-sivelestat group.

Abbreviations: bpm, beats per minute; ICU, intensive care unit; POD, postoperative days; CVP, central venous pressure; MOD, multiple organ dysfunction.

\section{Discussion}

Sivelestat contributed to early improvement in $\mathrm{PaO}_{2} / \mathrm{FiO}_{2}$, improvement in MOD score, and shorter periods of ventilator assistance and ICU stay for patients with ALI/ARDS following surgery for abdominal sepsis. Generally, selective neutrophil elastase inhibitors reduce pulmonary inflammation and improve pulmonary function. ${ }^{15}$ In a multicenter clinical study conducted in Japan, sivelestat contributed to early weaning from a ventilator in ALI/ARDS patients, resulting in early transfer to a general ward. ${ }^{6}$ However, in STRIVE (Sivelestat Trial in ALI Patients Requiring Mechanical Ventilation), no efficacy of sivelestat was found in patients with ALI/ARDS, even in the ventilator-assisted period, and there was no effect on mortality. ${ }^{7}$ One reason for the discrepant results between these two studies may be a difference in the severity of lung injury in the patients. ${ }^{16} \mathrm{In}$ addition, early ALI/ARDS is thought to be a condition for which sivelestat is likely to demonstrate efficacy. ${ }^{17}$ Herein, we studied the efficacy of sivelestat for ALI/ARDS during and immediately after surgery, because early diagnosis and therapeutic intervention for this condition are relatively easy. In addition, the APACHE II and SAPS II scores, which are indicators of extrapulmonary organ failure, did not differ significantly between patients who did and did not receive sivelestat, due to correction after intraoperative control. Significant reductions in the ventilator-assisted period and length of ICU stay in the sivelestat group may have occurred because the therapeutic intervention was performed in the early stage of lung injury, and was supported by real-time observation.

The highly heterogeneous causes of ALI/ARDS provide a further difficulty in studying the efficacy of sivelestat. ALI/ ARDS develops several hours after the onset of the systemic inflammatory response syndrome associated with sepsis. ${ }^{18}$ One of the primary target organs for neutrophil sequestration is the lung; thus, neutrophils are largely involved in the onset of ALI/ARDS associated with sepsis. ${ }^{19-21}$ All patients in this study had ALI/ARDS associated with abdominal sepsis, and thus neutrophil elastase inhibitors may have limited the progression of ALI/ARDS. ${ }^{22}$

Table 4 Patient outcome

\begin{tabular}{lllll}
\hline & Sivelestat & Non-sivelestat & P value & OR (95\% CI) \\
\hline Ventilator days & $6.6 \pm 6.1$ & $11.1 \pm 8.4$ & 0.034 & \\
Length of ICU stay (days) & $8.5 \pm 6.2$ & $13.3 \pm 9.5$ & 0.036 & 0.360 \\
ICU mortality, n (\%) & $2(6 \%)$ & $2(13 \%)$ & 0.380 & $0.052-3.198)$ \\
Inhospital mortality, n (\%) & $5(15 \%)$ & $4(27 \%)$ & 0.319 & $0.474(0.107-2.097)$ \\
\hline
\end{tabular}

Notes: Data are shown as the mean \pm standard deviation, or number (n) of patients; 34 patients in the sivelestat group, 15 patients in the non-sivelestat group. Abbreviations: $\mathrm{OR}$, odds ratio; $\mathrm{Cl}$, confidence interval; ICU, intensive care unit. 
The early improvement in MOD score in patients treated with sivelestat might be explained by improvement in $\mathrm{PaO}_{2} /$ $\mathrm{FiO}_{2}$ and maintenance of the platelet count. Neutrophil elastase promotes platelet aggregation, and thus sivelestat has antiplatelet activity and maintains the platelet count. ${ }^{23}$ The postoperative platelet count was maintained in the sivelestat group and was significantly higher than that in the non-sivelestat group. Furthermore, a decrease in platelet count after surgical invasion contributes to activation of neutrophils and endothelial cells. ${ }^{24}$ Therefore, the results of this study suggest that sivelestat inhibits activation of these cells by maintaining the platelet count, and thereby indirectly prevents onset and progression of ALI/ARDS.

The ventilation period and length of ICU stay decreased significantly in the sivelestat group. This result is consistent with the improvements seen in lung injury and MOD score. Because sivelestat is a neutrophil elastase inhibitor, its use in the early stages of ALI/ARDS is likely to be effective. In patients with ALI/ARDS associated with abdominal sepsis, onset of ALI/ARDS due to infection can be predicted during surgery, which makes it feasible to initiate sivelestat in the early stage after onset. This contributes to inhibition of excessive secretion of neutrophil elastase and neutrophil accumulation, thereby inhibiting further inflammation, ameliorating SIRS, and improving pulmonary function. ${ }^{25}$ In addition, platelet function is maintained, and activation of neutrophils and endothelial cells is inhibited, thereby allowing direct and indirect prevention of lung injury.

Control of excessive production of inflammatory cytokines may reduce surgical mortality. It has been shown that perioperative use of a neutrophil elastase inhibitor is beneficial for reducing the stress caused by invasive surgical procedures. Perioperative administration of sivelestat may reduce surgical stress by decreasing cytokine release and preserving antitumor immunity. ${ }^{26}$ Such modulation of excessive inflammatory cytokine production may improve surgical morbidity. Preoperative administration of a neutrophil elastase inhibitor has also been found to suppress increases in interleukin- 6 and to be useful for reducing surgical stress. ${ }^{27,28}$ However, the sample size was small in our study and this may have prevented demonstration of a significant improvement in mortality in the sivelestat group. A further study of the efficacy of sivelestat for ALI/ARDS would be desirable in a large-scale randomized clinical trial in patients with homogeneous baseline characteristics.

In conclusion, administration of sivelestat in the early stage of ALI/ARDS associated with abdominal sepsis contributes to early weaning from the ventilator and early discharge from ICU due to improvements in oxygenation potential and MOD score, as well as preservation of platelet function. Thus, early administration of sivelestat is likely to give a more successful therapeutic outcome for ALI/ARDS after surgery for abdominal sepsis.

\section{Disclosure}

The authors report no conflicts of interest in this work.

\section{References}

1. Abraham E, Matthay MA, Dinarello CA, et al. Consensus conference definitions for sepsis, septic shock, acute lung injury, and acute respiratory distress syndrome: time for a reevaluation. Crit Care Med. 2000; 28(1):232-235.

2. Spragg RG, Lewis JF, Walmrath HD, et al. Effect of recombinant surfactant protein C-based surfactant on the acute respiratory distress syndrome. N Engl J Med. 2004;351(9):884-892.

3. The ARDS Clinical Trials Network. Randomized, placebo-controlled trial of lisofylline for early treatment of acute lung injury and acute respiratory distress syndrome. Crit Care Med. 2002;30(1):1-6.

4. The ARDS Network. Ketoconazole for early treatment of acute lung injury and acute respiratory distress syndrome: a randomized controlled trial. JAMA. 2000;283(15):1995-2002.

5. Takeda S, Ishizaka A, Fujino Y, et al. Time to change diagnostic criteria of ARDS: towards the disease entity-based subgrouping. Pulm Pharmacol Ther. 2005;18(2):115-119.

6. Tamakuma S, Ogawa M, Aikawa N, et al. Relationship between neutrophil elastase and acute lung injury in humans. Pulm Pharmacol Ther. 2004;17(5):271-279.

7. Zeiher BG, Artigas A, Vincent JL, et al. Neutrophil elastase inhibition in acute lung injury: results of the STRIVE study. Crit Care Med. 2004; 32(8):1695-1702.

8. Bersten AD, Edibam C, Hunt T, Moran J. Incidence and mortality of acute lung injury and the acute respiratory distress syndrome in three Australian states. Am J Respir Crit Care Med. 2002;165(4): 443-448.

9. McGuire WW, Spragg RG, Cohen AB, Cochrane CG. Studies on the pathogenesis of the adult respiratory distress syndrome. J Clin Invest. 1982;69(3):543-553.

10. Guice KS, Oldham KT, Johnson KJ, Kunkel RG, Morganroth ML, Ward PA. Pancreatitis-induced acute lung injury. An ARDS model. Ann Surg. 1988;208(1):71-77.

11. Bernard GR, Artigas A, Brigham KL, et al. The American-European Consensus Conference on ARDS. Definitions, mechanisms, relevant outcomes, and clinical trial coordination. Am J Respir Crit Care Med. 1994;149(3 Pt 1):818-824.

12. Bone RC, Balk RA, Cerra FB, et al. Definitions for sepsis and organ failure and guidelines for the use of innovative therapies in sepsis. The ACCP/SCCM Consensus Conference Committee. American College of Chest Physicians/Society of Critical Care Medicine. Chest. 1992;101(6):1644-1655.

13. Marshall JC, Cook DJ, Christou NV, Bernard GR, Sprung CL, Sibbald WJ. Multiple organ dysfunction score: a reliable descriptor of a complex clinical outcome. Crit Care Med. 1995;23(10):1638-1652.

14. THE ARDS Network. Ventilation with lower tidal volumes as compared with traditional tidal volumes for acute lung injury and the acute respiratory distress syndrome. The Acute Respiratory Distress Syndrome Network. N Engl J Med. 2000;342(18):1301-1308.

15. Zeiher BG, Matsuoka S, Kawabata K, Repine JE. Neutrophil elastase and acute lung injury: prospects for sivelestat and other neutrophil elastase inhibitors as therapeutics. Crit Care Med. 2002;30(Suppl 5): S281-S287. 
16. Iwata K, Doi A, Ohji G, et al. Effect of neutrophil elastase inhibitor (sivelestat sodium) in the treatment of acute lung injury (ALI) and acute respiratory distress syndrome (ARDS): a systematic review and meta-analysis. Intern Med. 2010;49(22):2423-2432.

17. Endo S, Sato N, Yaegashi Y, et al. Sivelestat sodium hydrate improves septic acute lung injury by reducing alveolar dysfunction. Res Commun Mol Pathol Pharmacol. 2006;119(1/6):53-65.

18. Ware LB, Matthay MA. The acute respiratory distress syndrome. N Engl J Med. 2000;342(18):1334-1349.

19. Lin X, Yang H, Sakuragi T, et al. Alpha-chemokine receptor blockade reduces high mobility group box 1 protein-induced lung inflammation and injury and improves survival in sepsis. Am J Physiol Lung Cell Mol Physiol. 2005;289(4):583-590.

20. Kitagawa Y, Van Eeden SF, Redenbach DM, et al. Effect of mechanical deformation on structure and function of polymorphonuclear leukocytes. J Appl Physiol. 1997;82(5):1397-1405.

21. Razavi HM, Wang le F, Weicker S, et al. Pulmonary neutrophil infiltration in murine sepsis: role of inducible nitric oxide synthase. Am J Respir Crit Care Med. 2004;170(3):227-233.

22. Iba T, Kidokoro A, Fukunaga M, Takuhiro K, Yoshikawa S, Sugimotoa K. Pretreatment of sivelestat sodium hydrate improves the lung microcirculation and alveolar damage in lipopolysaccharideinduced acute lung inflammation in hamsters. Shock. 2006;26(1): 95-98.
23. Nishina K, Mikawa K, Takao Y, Maekawa N, Shiga M, Obara H. ONO-5046, an elastase inhibitor, attenuates endotoxin-induced acute lung injury in rabbits. Anesth Analg. 1997;84(5):1097-1103.

24. Ono S, Tsujimoto H, Hiraki S, et al. Effects of neutrophil elastase inhibitor on progression of acute lung injury following esophagectomy. World J Surg. 2007;31(10):1996-2001.

25. Donnelly SC, Strieter RM, Kunkel SL, et al. Interleukin-8 and development of adult respiratory distress syndrome in at-risk patient groups. Lancet. 1993;341(8846):643-647.

26. Akamoto S, Okano K, Sano T, et al. Neutrophil elastase inhibitor (sivelestat) preserves antitumor immunity and reduces the inflammatory mediators associated with major surgery. Surg Today. 2007;37(5): 359-365.

27. Ono S, Aosasa S, Mochizuki H. Effects of a protease inhibitor on reduction of surgical stress in esophagectomy. Am J Surg. 1999;177(1): 78-82.

28. Schulze S, Sommer P, Bigler D, et al. Effect of combined prednisolone, epidural analgesia, and indomethacin on the systemic response after colonic surgery. Arch Surg. 1992;127(3):325-331.
Drug Design, Development and Therapy

\section{Publish your work in this journal}

Drug Design, Development and Therapy is an international, peerreviewed open-access journal that spans the spectrum of drug design and development through to clinical applications. Clinical outcomes, patient safety, and programs for the development and effective, safe, and sustained use of medicines are a feature of the journal, which

\section{Dovepress}

has also been accepted for indexing on PubMed Central. The manuscript management system is completely online and includes a very quick and fair peer-review system, which is all easy to use. Visit http://www.dovepress.com/testimonials.php to read real quotes from published authors.

Submit your manuscript here: http://www.dovepress.com/drug-design-development-and-therapy-journal 